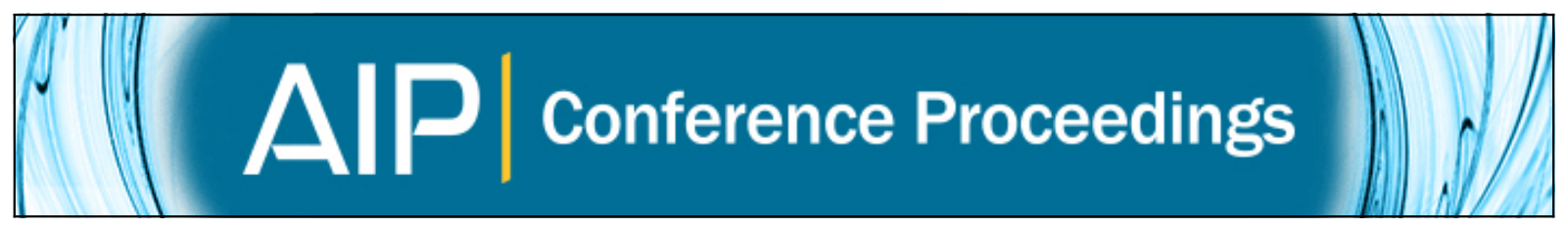

Relationship between solar energetic oxygen flux and MHD shock mach number

K. Liou, C. -C. Wu, M. Dryer, S. T. Wu, D. B. Berdichevsky, S. Plunkett, R. A. Mewaldt, and G. M. Mason

Citation: AIP Conference Proceedings 1436, 253 (2012); doi: 10.1063/1.4723616

View online: http://dx.doi.org/10.1063/1.4723616

View Table of Contents: http://scitation.aip.org/content/aip/proceeding/aipcp/1436?ver=pdfcov

Published by the AIP Publishing

Articles you may be interested in

Evolution of a magnetohydrodynamic coronal shock

AIP Conf. Proc. 1500, 50 (2012); 10.1063/1.4768744

Solar energetic particles: Shock acceleration and transport through self-amplified waves

AIP Conf. Proc. 1436, 212 (2012); 10.1063/1.4723610

Evolution of suprathermal seed particle and solar energetic particle abundances

AIP Conf. Proc. 1436, 206 (2012); 10.1063/1.4723609

Observations of the longitudinal spread of solar energetic particle events in solar cycle 24

AIP Conf. Proc. 1436, 103 (2012); 10.1063/1.4723596

Residual energy in MHD turbulence and in the solar wind

AIP Conf. Proc. 1436, 18 (2012); 10.1063/1.4723584 


\title{
Relationship Between Solar Energetic Oxygen Flux and MHD Shock Mach Number
}

\author{
K. Liou ${ }^{\mathrm{a}}$, C.-C. $\mathrm{Wu}^{\mathrm{b}}$, M. Dryer ${ }^{\mathrm{c}}$, S.T. Wu ${ }^{\mathrm{d}}$, D. B. Berdichevsky ${ }^{\mathrm{e}}$, S. \\ Plunkett $^{\mathrm{b}}$, R. A. Mewaldt ${ }^{\mathrm{f}}$, G. M. Mason ${ }^{\mathrm{a}}$ \\ ${ }^{a}$ Applied Physics Laboratory, Laurel, Maryland, USA \\ ${ }^{b}$ Naval Research Laboratory, Washington, DC, USA \\ ${ }^{c}$ NOAA Space Weather Prediction Center (ret.), Boulder, CO, USA \\ ${ }^{d}$ CSPAR, University of Alabama, Huntsville, Alabama, USA \\ ${ }^{e}$ Univ. of the District of Columbia, Washington, DC, USA \\ ${ }^{f}$ Caltech, Pasadena, CA, USA
}

\begin{abstract}
This study correlates the time-intensity profile of a magnetohydrodynamic (MHD) shock with the corresponding solar energetic oxygen for a coronal mass ejection (CME) event that occurred on October 28, 2003. The intensity of MHD shock, in terms of Mach number, is simulated using a 1.5D MHD code, whereas the solar energetic oxygen flux is observed by the Solar Isotope Spectrometer (SIS) on board the Advanced Composition Explorer (ACE) spacecraft. A good correlation (Pearson correlation coefficient: $r=0.70-0.84$ ) is found between the forward fast-mode shock Mach number and the hourly-averaged, logarithmic oxygen differential energy flux for 7 energy channels $(7.3-63.8 \mathrm{MeV})$. We suspect that the intensitytime profile of high energy SEP events is manifested by the strength (Mach number) of CMEdriven propagation shocks. While further studies with more events are required to be more conclusive, this study result provides a direction for future studies or predictions of SEP fluxes.
\end{abstract}

Keywords: Solar energetic particles, CME-driven shock, MHD simulation, ACE PACS: 95

\section{INTRODUCTION}

The finding that there is a close association of coronal mass ejection (CME) in "gradual" solar energetic particle (SEP) events has led to the belief that instead of solar flares, it is CME-driven shocks that are the source of the gradual SEPs [1,2]. Evidences for such an association also include but not limited to: (1) gradual SEP events are associated with large fluences and can last for days, corresponding to the transit time of CMEs in the inner heliosphere, (2) increase of particle fluxes associated with the passage of interplanetary shock [3], (3) a close association of type-II burst [4], (4) a good correlation between the peak intensity of SEP and the speed of CME [5], (5) SEP events are more likely for fast CMEs [6,7], and (6) observations of energetic ions upstream and downstream of the bow shock (a fast-mode shock) $[8,9,10]$. The successful prediction of the power-law spectrum of accelerated ions by the diffusive shock acceleration (DSA) theory [11] also favors such a belief.

While theories of shock acceleration have been developed and, to some extent, successfully tested in various situations [11,12], it is still difficult to predict the

Physics of the Heliosphere: A 10 Year Retrospective

AIP Conf. Proc. 1436, 253-258 (2012); doi: 10.1063/1.4723616

(C) 2012 American Institute of Physics 978-0-7354-1026-8/\$30.00 
intensity of SEP events. One of the key obstacles stems from observational constraints. The intensity-time profile of SEP events at 1 AU reflects the combined effect of shock strength, which controls acceleration processes, interplanetary medium, through which the CME-driven shock evolves and propagates, and the magnetic field structure that connects the SEP source region to the observational site. Successful modeling of SEP events will encounter and must address these difficulties.

An important parameter that has not been widely considered in SEP studies is the shock Mach number. The Mach number represents the strength of shock and is defined as the speed of a moving object divided by the anisotropic "sound speed" of the fluid. In the solar wind plasma, the "sound speed" is the velocity of fast-mode or slow-mode magnetohydrodynamic waves. To a first order approximation, the square of fast-mode shock Mach number is proportional to the kinetic energy of the plasma flow (in the shock frame) divided by the sum of the thermal and magnetic energy of the plasma. Thus it can be considered as a measure of free energy available for plasma heating downstream of the shock and for particle acceleration. Using one-dimensional hybrid simulations, Scholer et al. [13] demonstrate that higher Alfvén Mach number shocks result in flatter spectra. Therefore, it is reasonably to believe that a stronger (high Mach number) shock will produce more SEP particles.

In addition, the finding of a good correlation between ESP flux and the maximum CME speed reported by Kahler [5] may serve as "weak" evidence because a large (2-3 orders of magnitude) scatter appears in their SEP flux-CME speed relationship and because the CME speed is not the same as the Mach number. Our approach will have a number of simplifications: (a) we will use a 1.5D MHD model; (b) we will assume that the shock connection with the spacecraft is along the Sun-spacecraft line; and (c) we will limit our input simulation to only the major central meridian flare's CME's shock during a well-known Cycle 23 epoch. Here we will present the preliminary result of our case study that tests our view.

\section{DATA ANALYSIS}

In this study, we will examine one of the series of SEP events during the "Halloween" 2003 epoch [14]. Figure 1(a) shows hourly differential fluxes of energetic oxygen measurements made by the Solar Isotope Spectrometer (SIS) [15] on board the Advanced Composition Explorer (ACE) spacecraft on days 301 - 302 (October 28 - 29) of 2003. The use of the hourly data is to eliminate uncertainties caused by propagation of the energetic particles. For example, it takes less than $\sim 57$ min for $10 \mathrm{MeV} / \mathrm{n}$ oxygen particles to propagate to the Earth from the surface of Sun. There are eight energy channels, ranging from 7.3 to $89.8 \mathrm{MeV} / \mathrm{n}$, for oxygen. A sharp enhancement of the oxygen fluxes for all 8 energy channels was observed at $11 \mathrm{UT}$ of day 301, coinciding with the onset of a flare of X17.2 class (S16E08) [16]. The initial fast increase in the SEP fluxes was slowed $\sim 5$ hours after the onset and diminished a day later. There is a clear peak in the oxygen flux at the lowest energy channel $(7.30-9.99 \mathrm{MeV} / \mathrm{n})$. This peak occurs upon the arrival of an IP shock at $\sim 0600$ UT on day 302. Note that the oxygen fluxes did not drop to their pre-CME level. 


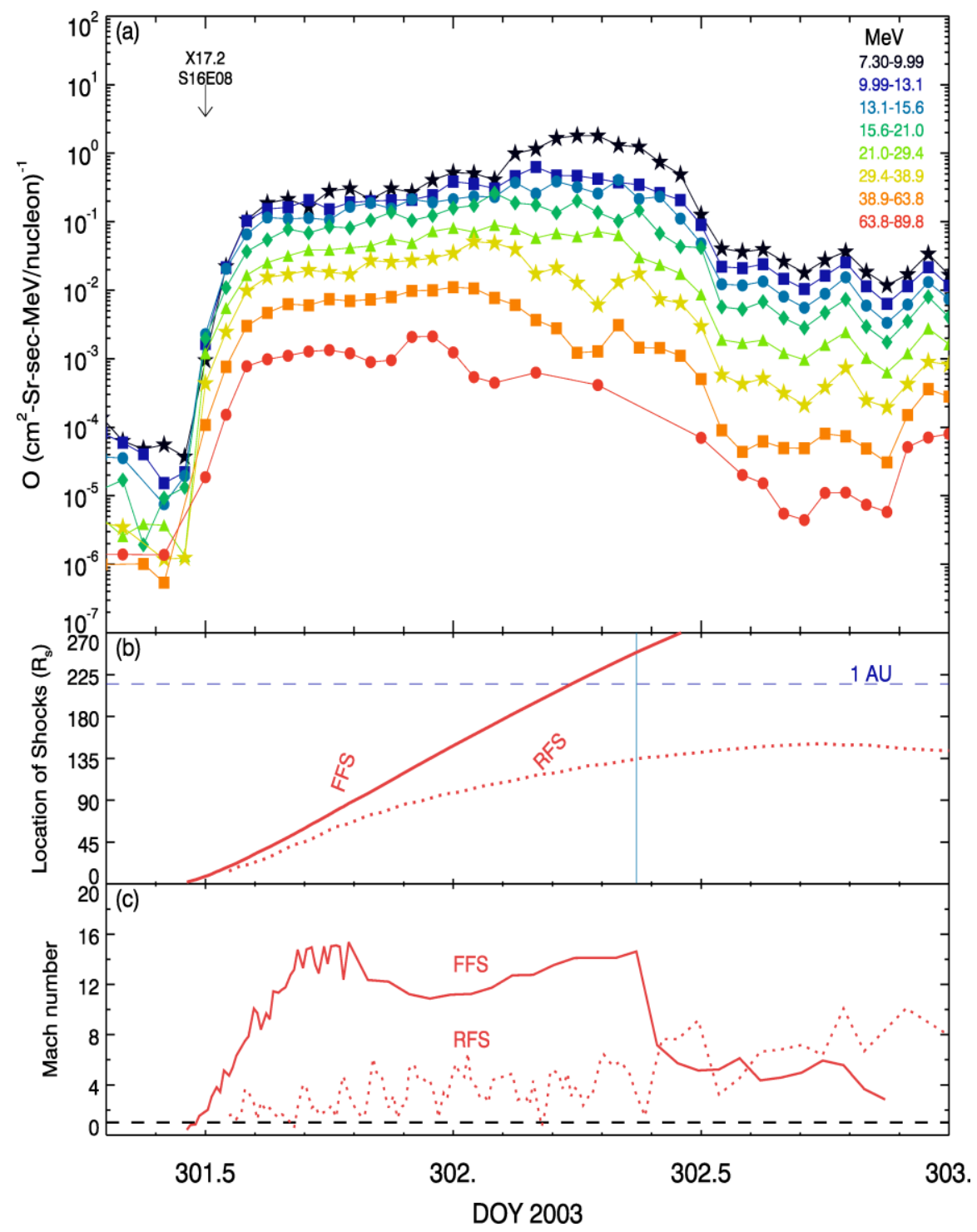

FIGURE 1. (a) Intensity-time profile of solar energetic oxygen observed by the Solar Isotope Spectrometer (SIS) on board the Advanced Composition Explorer (ACE) spacecraft at 8 energy channels: $7.3<\mathrm{E}<89.8 \mathrm{MeV} / \mathrm{n}$ during the period 28-29 October, 2003. The down-arrow indicates the time a solar flare of X17.2 in intensity is detected. (b) Distance of the simulated fast-mode shocks from the Sun. The vertical line indicates the time of shock arrival at the Earth. (c) Mach numbers for fastmode shock waves (FFS: fast forward shock, RFS: reverse forward shock). The simulation result is based on 1.5D MHD simulation results [18].

The enhancement of oxygen flux was apparently associated with the IP shock driven by the CME event associated with the X17.2 flare. Evolution of the CME and CME-driven shock for this particular event has been studied by Wu et al. [17-19], using a 1.5D adapted code [20]. The numerical code solves a set of MHD equations for a compressible, non-viscous, perfect conducting magnetohydrodynamic fluid in the presence of gravitational forces (from the Sun only). The input parameters (plasma density, temperature, and velocity associated with pressure pulses) were tuned to match the timing and the velocity profiles of the IP shock arriving at the ACE orbit. 
The fast-mode shock Mach numbers are obtained directly from their reported result and are used to correlate with the observed SEP fluxes. The use of the 1.5D approximation for the present study assumes that the shock origin is near the central meridian and that the magnetic connection to ACE is good; given the flare locations at E08, the assumption is reasonable.

Figure 1(b) shows the height-time profile of the simulated forward fast shock (FFS) and reverse fast shock (RFS). The simulation result suggests a constant speed for the fast forward shock (within $270 R_{s}$ ). However, the shock Mach number changes dramatically within the computational domain. As shown in Figure 1(c), the fast-mode shock Mach number $\left(\mathrm{M}_{\mathrm{F}}\right)$ increases linearly for the first $\sim 6$ hours up to $\mathrm{M}_{\mathrm{F}} \sim 16$. It sustains a high $(>12)$ Mach number shock for $\sim 17$ hours before it drops to $\mathrm{M}_{\mathrm{F}} \sim 6$. The Mach number for the reverse fast-mode shock shows a slow increase, with considerable fluctuations, while it is moving antisunward (note that the reverse shock propagates sunward but is carried out by the faster antisunward solar wind).
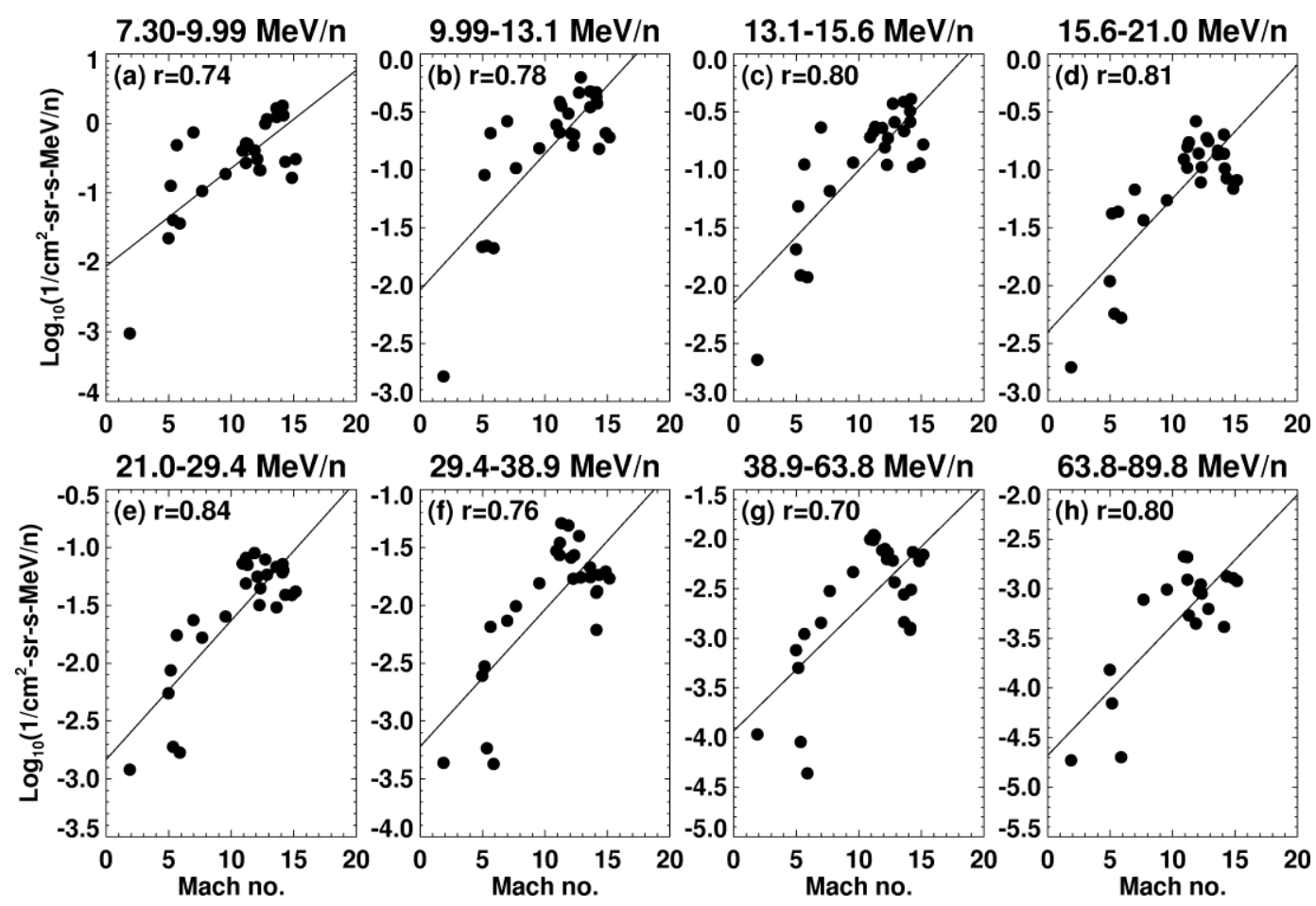

FIGURE 2. Scatter plots showing the relationship between the logarithmic hourly differential fluxes of energetic oxygen and fast-mode shock Mach numbers for 8 (from $\mathrm{a}-\mathrm{h}$ ) energy ranges. The Pearson correlation coefficient $(r)$ and linearly fitted line are provided in each panel tagged by the energy range in $\mathrm{MeV}$.

A comparison of the energetic oxygen fluxes with the fast-mode shock numbers suggests that a high degree of correlation between the Logarithmic oxygen differential fluxes and the forward (not the reverse) fast-mode Mach number. The scatter plots shown in Figure 2 demonstrate the relationship between the two hourly parameters. It 
is clearly shown that there is a trend that the oxygen flux increases with the $\mathrm{M}_{\mathrm{F}}$, regardless of the observed energy range. The Pearson correlation coefficient for each energy channel is calculated and it ranges from $0.7(38.9-63.8 \mathrm{MeV} / \mathrm{n})$ to $0.84(21.0$ $-29.4 \mathrm{MeV} / \mathrm{n}$ ). Using the autocorrelation analysis, we find the autocorrelation time is $\sim 3.5$ hours at the $95 \%$ significance. Given the number of data points $\mathrm{N}=27$ for all channels, except $\mathrm{N}=19$ for the highest energy channel, the independent number of the data points is $\sim 8$ ( 5 for the highest energy channel). The two-tail significance test suggests that the calculated correlation is at 95\% significance level (for $r=0.7$ and $\mathrm{N}$ $=8$ ) or better. For the highest energy channel, the independent number is less than 6 , and the significance cannot be assessed.

\section{DISCUSSION AND CONCLUSIONS}

In this study, we have explored the relationship between the intensity of solar energetic oxygen observed by ACE/SIS and the Mach number of a 1.5D numerically simulated CME-driven shock. We assumed a good magnetic field connection between the ACE and the source region and the source region must be large enough to cover the Sun-Earth line. This assumption is likely to be valid because the source of the solar flare, which is located at S16E08, is close to the central meridian. Moreover, Reames [21] suggested that the fast (> $2500 \mathrm{~km} / \mathrm{s}$ ) halo-CME can easily travel $2 R_{s}$ in 10 min where it could reach field lines well-connected to Earth and accelerate particle to high energy in time to be observed at $1 \mathrm{AU}$. We have also ignored a number of parameters that have been considered important in shock acceleration theories. These include, but not limited to, the shock type, determined by the angle between IMF and the shock normal, and the adiabatic deceleration. Under these assumptions, it is found that a good correlation exists between the time series oxygen differential flux and the time series of forward fast-mode shock Mach numbers for 7 out of 8 energy channels (7.3 63.8 MeV/n). It is thus suggested that fast-mode shock Mach numbers could be a controlling factor that manifests the intensity-time profile of SEPs with energies greater than $7.3 \mathrm{MeV} / \mathrm{n}$. The finding of a positive correlation between the Mach number of the fast-mode MHD shock and the SEP intensities is consistent with the general view that the shock strength is one of the key parameters that control the characteristics of SEP events. According to the diffusive shock acceleration theory, the power-law index of the spectrum of the accelerated particles depends solely on the shock's compression ratio. The Rankine-Hugoniot relation predicts that the shock compression ratio increases with the shock upstream Mach number and approaches $(\gamma+1) /(\gamma-1)$, where $\gamma$ is the heat capacity ratio, for a strong shock limit in hydrodynamic shocks [11]. For MHD shocks, the relation between the shock compression ratio and Mach number becomes more complex but the trend is similar [22]. Therefore, increasing shock Mach number may also harden particle distributions. This is consistent with hybrid simulation results [13]. One must keep in mind that the question about whether or not this scenario holds will require further study and be confirmed by observations.

To conclude, we have demonstrated, through a study of one large SEP event, that the time series of solar energetic ( $>7.3 \mathrm{MeV} / \mathrm{n}$ ) oxygen fluxes follows nicely with the

trend of the time series of forward fast-mode shock Mach numbers. This result 
suggests that the shock strength (Mach number) may be one of the parameters that control the intensity of SEP events.

\section{ACKNOWLEDGMENTS}

We acknowledge the use of ACE/SIS data, E. Stone is the principal investigator of the instrument, and the flare data from the NOAA Space Weather Prediction Center. We acknowledge support from NASA under contact NNN06AA01C (Task NNN10AA08T). The work of C.-C. Wu and S. Plunkett was supported by NRL ISES program. The work of S.-T Wu was supported by AFOSR Grant FA9550-07-1-0468 and NSF Grant ATM0754278 to UAH. The work of R. Mewaldt was supported by NASA Grant NNX08AI11G. The work of G. M. Mason was supported by NASA grants NNX10AT75G and NNX07AP69G.

\section{REFERENCES}

1. Kahler, S. W., E. W. Cliver, H. V. Cane., R. E. McGuire, R. G. Stone, and N. R. Sheeley, Jr., Astrophys. J., 302, 504 (1986).

2. Kahler, S. W., E. W. Cliver, H. V. Cane, R. E. McGuire, R. G. Stone, N. R. Sheeley, Jr., and R. A. Howard, Proc. 20th Int. Cosmic Ray Conf., Moscow, 3, 121 (1987).

3. Cane, H. V., T. T. von Rosenvinge, and R. E. McGuire, J. Geophys. Res., 95, 6575 (1990).

4. Cliver, E. W., S. W. Kahler, D. V. Reames, Astrophys. J., 605, 902 (2004).

5. Kahler, S. W., M. A. Shea, D. F. Smart, E. W. Cliver, in Proc. 22nd ICRC, 3, 21-24 (1991).

6. Reames, D. V., Space Sci. Rev., 90, 413-491 (1999).

7. Gopalswamy, N., in Proc. of the 20th National Solar Physics Meeting, Papradno, Slovakia, p. 108130 (2010).

8. Gosling, J. T., J. R. Asbridge, J. Bame, G. Pashmann, and N. Sckopke, Geophys. Res., Lett., 5, 957 (1978).

9. Ipavich, F. M., A. B. Galvin, G. Gloeckler, M. Scholer, and D. Hovestadt, J. Geophys. Res., 86, 4337 (1981).

10. Scholer, M. E. Mobius, L. M. Kistler, and B. Klecker, Geophys. Res., Lett., 16, 571-574 (1989).

11. Jones, F. C., and D. C. Ellison, Space Sci. Rev., 58, 259 (1991).

12. Zank, G. P., L. Gang, and O. Verkhoglyadova, Space Sci. Series ISSI, 27, 255-272 (2007).

13. Scholer, M. H. Kucharek, K.-H. Trattner, Ann. Geophys., 17, 583-594 (1999).

14. Dryer, M., Z. Smith, C. D. Fry, W. Sun, C. S. Deehr and S.-I. Akasofu, Space Weather, 2, S09001, doi:10.1029/2004SW000087 (2004).

15. Stone, E. C., and et al., Space Sci. Rev., 86, 357-408 (1998).

16. Mewaldt, R. A., and et al., J. Geophys. Res., 110, A09S18, doi:10.2019/2005JA011038 (2005).

17. Wu, C.-C., et al., J. Geophys. Res., 110(A9), A09S17, doi:10.1029/2005JA011011 (2005).

18. Wu, C.-C., X.-S. Feng, S.-T. Wu, M. Dryer, C. D. Fry, J. Geophys. Res., 111(A12), A12104,10.1029/2006JA011615 (2006)

19. Wu, C.-C., S.-T. Wu, M. Dryer, C. D. Fry, D. Berdichevsky, Z. Smith, and T. Detman, J. Atmos. Solar-Terr. Phys., 69(1-2), 91-100 (2007).

20. Panitchob, S. (1987), Ph.D. Dissertation, Dept. of Mech. Eng., Univ. of Ala., Huntsville, Ala. (1987).

21. Reames, D. V., Astrophys. J., 693, 812-821 (2009).22. Russell, C. T., in Collisionless Shocks in the Heliosphere: Reviews of Current Research, AGU Geophys. Monogr., 35, p.109 (1985). 